\title{
Synthesis and Antiviral Activity of Hydrogenated Ferulic Acid Derivatives
}

\author{
Can Cui, ${ }^{1}$ Zhi-Peng Wang, ${ }^{1}$ Xiu-jiang Du, ${ }^{1}$ Li-Zhong Wang, ${ }^{1}$ Shu-Jing Yu, ${ }^{1}$ Xing-Hai Liu, ${ }^{2}$ \\ Zheng-Ming Li, ${ }^{1}$ and Wei-Guang Zhao ${ }^{1}$ \\ ${ }^{1}$ State Key Laboratory of Elemento-Organic Chemistry, National Pesticide Engineering Research Center (Tianjin), Nankai University, \\ Tianjin 300071, China \\ ${ }^{2}$ College of Chemical Engineering \& Materials Sciences, Zhejiang University of Technology, Hangzhou 310014, China
}

Correspondence should be addressed to Xing-Hai Liu; xhliu@zjut.edu.cn and Wei-Guang Zhao; zwg@nankai.edu.cn

Received 23 January 2013; Accepted 19 March 2013

Academic Editor: Alberto Ritieni

Copyright (C) 2013 Can Cui et al. This is an open access article distributed under the Creative Commons Attribution License, which permits unrestricted use, distribution, and reproduction in any medium, provided the original work is properly cited.

A series of hydrogenated ferulic acid amide derivatives 4 were synthesized. The molecular structures of the synthesized compounds were analyzed by ${ }^{1} \mathrm{H}$ NMR and HRMS. The biological activity study showed that some of them displayed excellent protection activity and curative activity against TMV at $500 \mu \mathrm{g} / \mathrm{mL}$.

\section{Introduction}

Viruses are among the most agriculturally important groups of plant pathogens, causing serious economic losses in many major crops by reducing yield and quality [1]. Although plant viruses are genetically rather simple, they are difficult to prevent or control and have devastating impact on crop growth. So, plant virus is often referred to as "plant cancer" for several reasons. It is reported that three class pesticides are discovered. For example, nucleoside medicines as ribavirin, tiazofurin, selenazofurin, and benzamide riboside, nonnucleosides such as dufulin and mycophenolic acid, and natural product as ningnanmycin can inhibit virus replication effectively and suppress virus symptoms.

Ferulic acid is isolated from many staple foods, such as fruits, vegetables, cereals, and coffee. It and its derivatives exhibited diversity activity, such as anticancer $[2,3]$, antiatherogenic [4], anticarcinogenic [5], and antibacterial agents [6], as well as anti-inflammatory activity $[7,8]$. In view of these facts mentioned earlier, and also as a part of our work on the synthesis of bioactive lead compounds for drug discovery, the title compounds were designed. We herein report a series of hydrogenated ferulic acid amide derivatives 4 that have been found to possess potent antiviral activities.

\section{Results and Discussion}

2.1. Chemistry. All of the hydrogenated ferulic acid amide derivatives were prepared from a key intermediate hydrogenated ethyl ferulate, which was easily prepared by catalytic hydrogenation of the ferulic acid 1 using $\mathrm{Pd} / \mathrm{C}$ and $\mathrm{H}_{2}$ ( $1 \mathrm{~atm})$, as shown in Scheme 1. Traditional methods of synthesis of amide suffer from disadvantages, such as long reaction time and inconvenience of handling. In this paper, an eco-friendly and high-yielding method for hydrogenated ferulic acid amides $\mathbf{3}$ from hydrogenated ethyl ferulate $\mathbf{2}$ and substituted 2-amino-1-phenylethanol was applied. It can be obtained under microwave irradiation at $130^{\circ} \mathrm{C}$ in freesolvent conditions for $30 \mathrm{~min}$ in generally good yields. As was mentioned earlier, alkylation of the compounds 3 yielded the compounds 4 followed by alkylation with bromoalkane to give the $\mathrm{N}$-(2-alkoxy-2-phenylethyl) hydrogenated ferulic acid amide derivatives 4 .

2.2. Spectrum. In the ${ }^{1} \mathrm{H}$ NMR spectra of title compounds, the $\mathrm{NH}$ proton signals of title compounds were appeared at $\delta 5.81 \sim 6.02 \mathrm{ppm}$. All the alkyl or aryl groups showed the normal location. All the title compounds of HRMS matched with the theoretical values. 
TABLE 1: Antiviral activities of the title compounds at $500 \mu \mathrm{g} / \mathrm{mL}$.

\begin{tabular}{lcccc}
\hline Compound & \multirow{2}{*}{$\mathrm{R}_{1}$} & $\mathrm{R}_{2}$ & $\begin{array}{c}\text { Anti-TMV activities } \\
\text { Protection } \\
\text { effect }\end{array}$ & $\begin{array}{c}\text { Curative } \\
\text { effect }\end{array}$ \\
\hline $\mathbf{4 a}$ & $m-\mathrm{MeO}$ & Benzyl & 0 & 24.2 \\
$\mathbf{4 b}$ & $m-\mathrm{NO}_{2}$ & Benzyl & 10.4 & 0 \\
$\mathbf{4 c}$ & $m-\mathrm{NO}_{2}$ & Propynyl & 0 & 0 \\
$\mathbf{4 d}$ & $p$ - $\mathrm{NO}_{2}$ & Benzyl & 0 & 0 \\
$\mathbf{4 e}$ & $p$-benzyloxy & Benzyl & 0 & 12.3 \\
$\mathbf{4 f}$ & $p$-benzyloxy & Propynyl & 15.2 & 28.9 \\
$\mathbf{4 g}$ & $p$-benzyloxy & Allyl & 22.8 & 20.7 \\
Ribavirin & & & 32.6 & 38.5 \\
\hline
\end{tabular}

2.3. Antiviral Activity. To make a judgment of the antiviral potency of the title compounds, the commercially available plant virucide ribavirin was used as the control. The in vivo antiviral results of all the title compounds against TMV are listed in Table 1 . The results showed that title compounds exhibited varying degrees of activities against TMV. Compound $\mathbf{4 g}$ displayed good protection activity $(22.8 \%)$ at $500 \mu \mathrm{g} / \mathrm{mL}$, which is the same of ribavirin at $500 \mu \mathrm{g} / \mathrm{mL}$ (32.6\%). Compounds $\mathbf{4 a}$ and $\mathbf{4 f}$ showed the same curative activity level $(24.2 \%$ and $28.9 \%$, resp.) as ribavirin at $500 \mu \mathrm{g} / \mathrm{mL}(32.6 \%)$.

\section{Experimental}

3.1. Materials and Methods. ${ }^{1} \mathrm{H}$ NMR spectra were measured on a Bruker AC-P500 instrument using TMS as an internal standard and $\mathrm{CDCl}_{3}$ as solvent. Melting points were determined on an X-4 binocular microscope melting point apparatus (Beijing Tech Instruments Co., Beijing, China) and were uncorrected. HRMS were recorded on an IonSpec 7.0 T Fourier Transform Ion Cyclotron Resonance (FTICR) Mass Spectrometer. All chemicals or reagents were purchased from standard commercial suppliers.

3.2. Synthesis. All of the hydrogenated ferulic acid amide derivatives were prepared as shown in Scheme 1.

To a solution of 3-(3,4-dimethoxyphenyl)acrylic acid $(15 \mathrm{~g}, 72 \mathrm{mmol})$ in ethyl acetate $(150 \mathrm{~mL})$ and ethanol $(150 \mathrm{~mL}), \mathrm{Pd} / \mathrm{C}(1.5 \mathrm{~g}, 10 \%)$ was added under the $\mathrm{N}_{2}$ atmosphere. The mixture was refluxed for $72 \mathrm{~h}$. After the reaction was completed, the $\mathrm{Pd} / \mathrm{C}$ was filtered, and the solvent was evaporated. The combined organic phases were washed with brine, dried over $\mathrm{MgSO}_{4}$, and evaporated to afford white solid, yield $92 \%$, m.p. $92-94^{\circ} \mathrm{C}$ (ref. $96-97^{\circ} \mathrm{C}$ ).

To a solution of 3-(3,4-dimethoxyphenyl)propanoic acid $(1.44 \mathrm{~g}, 6.84 \mathrm{mmol})$ in THF under $-20^{\circ} \mathrm{C}$, isobutyl chloroformate $(0.89 \mathrm{~g}, 6.84 \mathrm{mmol})$ and NMM $(0.7 \mathrm{~g}, 7.18 \mathrm{mmol})$ were added. The mixture was stirred for $30 \mathrm{~min}$. A solution of 2-amino-1-(4-nitrophenyl)ethanol $(7.18 \mathrm{mmol})$ in THF was dropwise in the mixture, then it was stirred for $12 \mathrm{~h}$ at room temperature. After the reaction was completed, the THF was evaporated, and the residue was diluted with water and extracted several times with dichloromethane. The combined organic phases were washed with brine, dried over $\mathrm{MgSO}_{4}$, and evaporated. The remainder was purified by chromatography on silica gel using petroleum ether (60$90^{\circ} \mathrm{C}$ ) and ethyl acetate as the eluent to afford compound 3.

3-(3,4-Dimethoxyphenyl)-N-(2-hydroxy-2-(3-methoxyphenyl) ethyl)propanamide $3 a$. Yellow solid, yield 85\%, m.p. 112$113^{\circ} \mathrm{C},{ }^{1} \mathrm{H}$ NMR $\left(400 \mathrm{MHz}, \mathrm{CDCl}_{3}\right) \delta 2.49(\mathrm{~d}, J=7.7 \mathrm{~Hz}$, $2 \mathrm{H}, \mathrm{CH}_{2} \mathrm{CH}_{2}$ ), 2.91 (t, $\left.J=7.7 \mathrm{~Hz}, 2 \mathrm{H}, \mathrm{CH}_{2} \mathrm{CH}_{2}\right), 3.32(\mathrm{~s}, 1 \mathrm{H}$, $\mathrm{ArCHCH}_{2}$ ), 3.69 (s, 1H, $\mathrm{ArCHCH}_{2}$ ), 3.83 (s, 9H, p- $\mathrm{CH}_{3} \mathrm{O}$ $\left.+p-\mathrm{CH}_{3} \mathrm{O}+p-\mathrm{CH}_{3} \mathrm{O}\right), 4.77(\mathrm{~s}, 1 \mathrm{H}, \mathrm{ArCH}), 5.80$ (br, $1 \mathrm{H}$, CONH), 7.01-6.67 (m, 6H, Ar-H).

\section{3-(3,4-Dimethoxyphenyl)-N-(2-hydroxy-2-(3-nitrophenyl)} ethyl)propanamide $3 \boldsymbol{b}$. Yellow oil, yield $82 \%,{ }^{1} \mathrm{H}$ NMR $\left(400 \mathrm{MHz}, \mathrm{CDCl}_{3}\right) \delta 2.50\left(\mathrm{t}, J=7.6 \mathrm{~Hz}, 2 \mathrm{H}, \mathrm{CH}_{2} \mathrm{CH}_{2}\right), 2.90$ (t, $\left.J=7.6 \mathrm{~Hz}, 2 \mathrm{H}, \mathrm{CH}_{2} \mathrm{CH}_{2}\right), 3.30-3.36\left(\mathrm{~m}, 1 \mathrm{H}, \mathrm{CH}_{2} \mathrm{CH}\right)$, 3.63-3.68 (m, $\left.1 \mathrm{H}, \mathrm{CH}_{2} \mathrm{CH}\right), 3.85\left(\mathrm{~s}, 6 \mathrm{H}, p-\mathrm{CH}_{3} \mathrm{O}+o-\mathrm{CH}_{3} \mathrm{O}\right)$, $4.90\left(\mathrm{dd}, J=2.4 \mathrm{~Hz}, J=7.2 \mathrm{~Hz}, 1 \mathrm{H}, \mathrm{CH}_{2} \mathrm{CH}\right), 6.03(\mathrm{br}, 1 \mathrm{H}$, CONH), 6.70-6.79 (m, 3H, Ar-H), 7.63-8.20 (m, 4H, Ar-H).

3-(3,4-Dimethoxyphenyl)-N-(2-hydroxy-2-(4-nitrophenyl) ethyl)propanamide 3c. White solid, yield 51\%, m.p. 133$135^{\circ} \mathrm{C},{ }^{1} \mathrm{H}$ NMR $\left(400 \mathrm{MHz}, \mathrm{CDCl}_{3}\right) \delta 2.47-2.52(\mathrm{~m}, 2 \mathrm{H}$, $\left.\mathrm{CH}_{2} \mathrm{CH}_{2}\right), 2.92\left(\mathrm{t}, \mathrm{J}=7.2 \mathrm{~Hz}, 2 \mathrm{H}, \mathrm{CH}_{2} \mathrm{CH}_{2}\right), 3.26-3.32$ (m, $\left.1 \mathrm{H}, \mathrm{CH}_{2} \mathrm{CH}\right), 3.66-3.71\left(\mathrm{~m}, 1 \mathrm{H}, \mathrm{CH}_{2} \mathrm{CH}\right), 3.87(\mathrm{~s}, 6 \mathrm{H}$, $\left.p-\mathrm{CH}_{3} \mathrm{O}+o-\mathrm{CH}_{3} \mathrm{O}\right), 4.91(\mathrm{dd}, J=2.4 \mathrm{~Hz}, J=6.8 \mathrm{~Hz}, 1 \mathrm{H}$, $\left.\mathrm{CH}_{2} \mathrm{CH}\right), 5.73$ (s, 1H, CONH), 6.71-6.81 (m, 3H, Ar-H), 7.45 (d, $J=8.8 \mathrm{~Hz}, 2 \mathrm{H}, \mathrm{Ar}-\mathrm{H}), 8.17$ (d, $J=8.8 \mathrm{~Hz}, 1 \mathrm{H}, \mathrm{Ar}-\mathrm{H})$.

N-(2-(4-(Benzyloxy)phenyl)-2-hydroxyethyl)-3-(3,4-dimethoxyphenyl)propanamide $3 d$. Yellow solid, yield 67\%, m.p. $132-133^{\circ} \mathrm{C},{ }^{1} \mathrm{H}$ NMR $\left(400 \mathrm{MHz}, \mathrm{CDCl}_{3}\right) \delta 2.45(\mathrm{t}, J=7.5 \mathrm{~Hz}$, $\left.2 \mathrm{H}, \mathrm{CH}_{2} \mathrm{CH}_{2}\right), 2.91\left(\mathrm{t}, J=7.4 \mathrm{~Hz}, 2 \mathrm{H}, \mathrm{CH}_{2} \mathrm{CH}_{2}\right), 3.24(\mathrm{t}$, $\left.J=11.2 \mathrm{~Hz}, 1 \mathrm{H}, \mathrm{ArCHCH}_{2}\right), 3.80-3.66\left(\mathrm{~m}, 1 \mathrm{H}, \mathrm{ArCHCH}_{2}\right)$, $3.88\left(\mathrm{~s}, 6 \mathrm{H}, p-\mathrm{CH}_{3} \mathrm{O}+o-\mathrm{CH}_{3} \mathrm{O}\right), 4.22(\mathrm{~d}, J=11.5 \mathrm{~Hz}$, $\left.1 \mathrm{H}, \mathrm{ArCHCH}_{2}\right), 4.37\left(\mathrm{~d}, J=8.7 \mathrm{~Hz}, 1 \mathrm{H}, \mathrm{ArCH}_{2}\right), 4.48(\mathrm{~d}$, $\left.J=11.6 \mathrm{~Hz}, 1 \mathrm{H}, \mathrm{ArCHCH}_{2}\right), 5.10\left(\mathrm{~s}, 2 \mathrm{H}, \mathrm{ArCH}_{2}\right), 5.86(\mathrm{br}$, $1 \mathrm{H}, \mathrm{CONH}), 6.84-6.69(\mathrm{~m}, 3 \mathrm{H}, \mathrm{Ar}-\mathrm{H}), 7.53-7.22(\mathrm{~m}, 9 \mathrm{H}$, Ar- $\mathrm{H})$.

RX (7.56 mmol) was added to a mixture of $3(3.78 \mathrm{mmol})$, $30 \%$ aqueous sodium hydroxide solution $(18.9 \mathrm{mmol})$, and catalytic amount of hexadecyl trimethyl ammonium bromide $(0.4 \mathrm{mmol})$ in $6 \mathrm{~mL}$ of $\mathrm{CH}_{2} \mathrm{Cl}_{2}$ at room temperature. The reaction mixture was heated at reflux for $8 \mathrm{~h}$. Subsequently, the mixture was diluted with water and extracted several times with dichloromethane. The combined organic phases were washed with brine, dried over $\mathrm{MgSO}_{4}$, and evaporated. The remainder was purified by chromatography on silica gel using petroleum ether $\left(60-90^{\circ} \mathrm{C}\right)$ and ethyl acetate as the eluent to afford the title compounds $\mathbf{4 a - 4 g}$.

N-(2-(Benzyloxy)-2-(3-methoxyphenyl)ethyl)-3-(3,4-dimethoxyphenyl)propanamide $4 a$. Yellow oil, yield $85 \%,{ }^{1} \mathrm{H}$ NMR $\left(400 \mathrm{MHz} \mathrm{CDCl}_{3}\right) \delta 2.42\left(\mathrm{t}, J=7.7 \mathrm{~Hz}, 2 \mathrm{H}, \mathrm{CH}_{2} \mathrm{CH}_{2}\right), 2.88$ (t, $\left.J=7.7 \mathrm{~Hz}, 2 \mathrm{H}, \mathrm{CH}_{2} \mathrm{CH}_{2}\right), 3.25-3.16\left(\mathrm{~m}, 1 \mathrm{H}, \mathrm{ArCHCH}_{2}\right)$, 3.77-3.69 (m, 1H, ArCHCH 2$), 3.87-3.80\left(\mathrm{~m}, 9 \mathrm{H}, p-\mathrm{CH}_{3} \mathrm{O}\right.$ $\left.+p-\mathrm{CH}_{3} \mathrm{O}+o-\mathrm{CH}_{3} \mathrm{O}\right), 4.22(\mathrm{~d}, \mathrm{~J}=11.6 \mathrm{~Hz}, 1 \mathrm{H}, \mathrm{ArCH})$, 

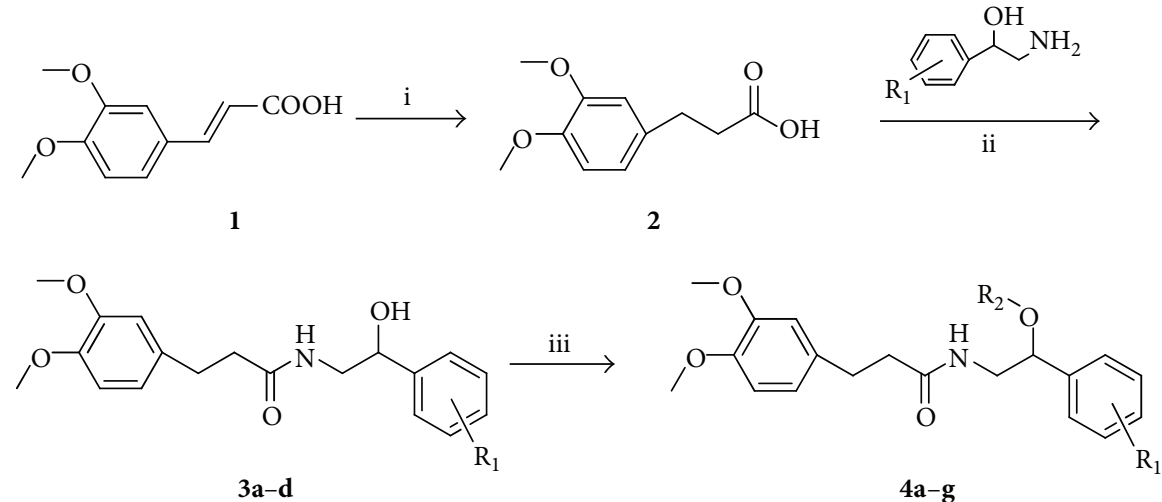

Scheme 1: Reagents and conditions: (i) $10 \% \mathrm{Pd} / \mathrm{C}, \mathrm{H}_{2}$, EtOH, EA; (ii) isobutyl chloroformate, NMM; (iii) $\mathrm{R}_{1} \mathrm{Br}, \mathrm{TBAB}, \mathrm{NaOH}, 65^{\circ} \mathrm{C}$.

$4.38\left(\mathrm{dd}, J=8.8 \mathrm{~Hz}, J=3.7 \mathrm{~Hz}, 1 \mathrm{H}, \mathrm{ArCHCH}_{2}\right), 4.49(\mathrm{~d}$, $J=11.6 \mathrm{~Hz}, 1 \mathrm{H}, \mathrm{ArCH}), 5.81(\mathrm{~s}, 1 \mathrm{H}, \mathrm{CONH}), 6.80-6.70$ (m, 3H, Ar-H), 6.87-6.81 (m, 3H, Ar-H), 7.38-7.24 (m, 5H, Ar-H). HRMS (ESI) m/z Calcd for $\mathrm{C}_{27} \mathrm{H}_{31} \mathrm{NO}_{5} \mathrm{Na}^{+}[\mathrm{M}+\mathrm{Na}]^{+}$ 472.2094, found 472.2091.

N-(2-(Benzyloxy)-2-(3-nitrophenyl)ethyl)-3-(3,4-dimethoxyphenyl)propanamide $4 \boldsymbol{b}$. Yellow oil, yield $82 \%,{ }^{1} \mathrm{H}$ NMR $\left(400 \mathrm{MHz}, \mathrm{CDCl}_{3}\right) \delta 2.44\left(\mathrm{t}, J=7.6 \mathrm{~Hz}, 2 \mathrm{H}, \mathrm{CH}_{2} \mathrm{CH}_{2}\right), 2.88$ $\left(\mathrm{t}, J=7.6 \mathrm{~Hz}, 2 \mathrm{H}, \mathrm{CH}_{2} \mathrm{CH}_{2}\right), 3.28-3.22\left(\mathrm{~m}, 1 \mathrm{H}, \mathrm{CH}_{2} \mathrm{CH}\right)$, 3.75-3.72 (m, $\left.1 \mathrm{H}, \mathrm{CH}_{2} \mathrm{CH}\right), 3.86\left(\mathrm{~s}, 3 \mathrm{H}, o-\mathrm{CH}_{3} \mathrm{O}\right), 3.88(\mathrm{~s}$, $\left.3 \mathrm{H}, p-\mathrm{ArOCH}_{3}\right), 4.28(\mathrm{~d}, J=11.5 \mathrm{~Hz}, 1 \mathrm{H}, \mathrm{Ar}-\mathrm{CH}), 4.51$ (d, $J=11.5 \mathrm{~Hz}, 1 \mathrm{H}, \mathrm{Ar}-\mathrm{CH}), 4.56\left(\mathrm{~d}, J=8.0 \mathrm{~Hz}, 1 \mathrm{H}, \mathrm{CH}_{2} \mathrm{CH}\right)$, 5.81 (br, $1 \mathrm{H}, \mathrm{CONH}), 6.84-6.72(\mathrm{~m}, 3 \mathrm{H}, \mathrm{Ar}-\mathrm{H}), 7.43-7.32(\mathrm{~m}$, $4 \mathrm{H}, \mathrm{Ar}-\mathrm{H}), 7.57$ (t, $J=8.0 \mathrm{~Hz}, 1 \mathrm{H}, \mathrm{Ar}-\mathrm{H}), 7.67$ (d, $J=8.0 \mathrm{~Hz}$, $1 \mathrm{H} \mathrm{Ar}-\mathrm{H}), 8.21$ (d, J = 8.0 Hz, 1H, Ar-H), 8.27 (s, 1H, Ar-H). HRMS (ESI) $\mathrm{m} / \mathrm{z}$ Calcd for $\mathrm{C}_{26} \mathrm{H}_{28} \mathrm{~N}_{2} \mathrm{O}_{6} \mathrm{Na}^{+}[\mathrm{M}+\mathrm{Na}]^{+}$ 487.1840, found 487.1835 .

3-(3,4-Dimethoxyphenyl)-N-(2-(3-nitrophenyl)-2-(prop-2yn-1-yloxy)ethyl)propanamide 4c. Yellow oil, yield 95\%, ${ }^{1} \mathrm{H}$ NMR $\left(400 \mathrm{MHz}, \mathrm{CDCl}_{3}\right) \delta 2.40\left(\mathrm{~m}, 3 \mathrm{H}, \mathrm{CH}_{2} \mathrm{CH}_{2}+\right.$ $\mathrm{C} \equiv \mathrm{CH}), 2.80\left(\mathrm{~d}, J=7.6 \mathrm{~Hz}, 2 \mathrm{H}, \mathrm{CH}_{2} \mathrm{CH}_{2}\right), 3.22-3.08(\mathrm{~m}$, $1 \mathrm{H}, \mathrm{ArCHCH}_{2}$ ), $3.66\left(\mathrm{~m}, 1 \mathrm{H}, \mathrm{ArCHCH}_{2}\right), 3.88-3.72(\mathrm{~m}, 7 \mathrm{H}$, $\left.p-\mathrm{CH}_{3} \mathrm{O}+o-\mathrm{CH}_{3} \mathrm{O}+\mathrm{OCH}_{2} \mathrm{CH}\right), 4.09\left(\mathrm{~m}, 1 \mathrm{H}, \mathrm{OCH}_{2} \mathrm{CH}\right)$, $4.64\left(\mathrm{dd}, J=3.0 \mathrm{~Hz}, J=8.0 \mathrm{~Hz}, 1 \mathrm{H}, \mathrm{ArCHCH}_{2}\right), 6.02(\mathrm{br}$, $1 \mathrm{H}, \mathrm{CONH}), 6.78-6.61(\mathrm{~m}, 3 \mathrm{H}, \mathrm{Ar}-\mathrm{H}), 7.46$ (s, 1H, Ar-H), 7.56 (s, 1H, Ar-H), 8.24-8.03 (m, 2H, Ar-H). HRMS (ESI) $\mathrm{m} / \mathrm{z}$ Calcd for $\mathrm{C}_{22} \mathrm{H}_{24} \mathrm{~N}_{2} \mathrm{O}_{6} \mathrm{Na}^{+}[\mathrm{M}+\mathrm{Na}]^{+}$435.1527, found 435.1526 .

N-(2-(Benzyloxy)-2-(4-nitrophenyl)ethyl)-3-(3,4-dimethoxyphenyl)propanamide 4 d. Yellow solid, yield 76.8\%, m.p. $133-135^{\circ} \mathrm{C},{ }^{1} \mathrm{H}$ NMR $\left(400 \mathrm{MHz}, \mathrm{CDCl}_{3}\right) \delta 2.43(\mathrm{t}, J=7.6 \mathrm{~Hz}$, $2 \mathrm{H}, \mathrm{CH}_{2} \mathrm{CH}_{2}$ ), 2.87 ( $\mathrm{t}, \mathrm{J}=7.6 \mathrm{~Hz}, 2 \mathrm{H}, \mathrm{CH}_{2} \mathrm{CH}_{2}$ ), 3.19-3.26 (m, $\left.1 \mathrm{H}, \mathrm{CH}_{2} \mathrm{CH}\right), 3.67-3.73\left(\mathrm{~m}, 1 \mathrm{H}, \mathrm{CH}_{2} \mathrm{CH}\right), 3.83(\mathrm{~s}, 3 \mathrm{H}$, $\left.o-\mathrm{ArOCH}_{3}\right), 3.85\left(\mathrm{~s}, 3 \mathrm{H}, p-\mathrm{ArOCH}_{3}\right), 4.26(\mathrm{~d}, J=11.6 \mathrm{~Hz}$, $1 \mathrm{H}, \operatorname{Ar}-\mathrm{CH}), 4.47(\mathrm{~d}, J=11.6 \mathrm{~Hz}, 1 \mathrm{H}, \mathrm{Ar}-\mathrm{CH}), 4.54(\mathrm{dd}$, $\left.J=4 \mathrm{~Hz}, J=8 \mathrm{~Hz}, 1 \mathrm{H}, \mathrm{CH}_{2} \mathrm{CH}\right), 5.86$ (br, $\left.1 \mathrm{H}, \mathrm{CONH}\right)$, 6.71-6.78 (m, 3H, Ar-H), 7.25-7.38 (m, 5H, Ar-H), 7.50 (d, $J=8.4 \mathrm{~Hz}, 2 \mathrm{H}, \mathrm{Ar}-\mathrm{H}), 8.21$ (d, $J=8.4 \mathrm{~Hz}, 2 \mathrm{H}, \mathrm{Ar}-\mathrm{H})$. HRMS
(ESI) $\mathrm{m} / \mathrm{z}$ Calcd for $\mathrm{C}_{26} \mathrm{H}_{28} \mathrm{~N}_{2} \mathrm{O}_{6} \mathrm{Na}^{+}[\mathrm{M}+\mathrm{Na}]^{+}$487.1840, found 487.1836 .

N-(2-(Benzyloxy)-2-(4-(benzyloxy)phenyl)ethyl)-3-(3,4-dimethoxyphenyl)propanamide $4 \boldsymbol{e}$. White solid, yield 68\%, m.p. $107-108^{\circ} \mathrm{C},{ }^{1} \mathrm{H}$ NMR $\left(400 \mathrm{MHz}, \mathrm{CDCl}_{3}\right) \delta 2.45(\mathrm{t}, J=8.0 \mathrm{~Hz}$, $2 \mathrm{H}, \mathrm{CH}_{2} \mathrm{CH}_{2}$ ), $2.92\left(\mathrm{t}, \mathrm{J}=8.0 \mathrm{~Hz}, 2 \mathrm{H}, \mathrm{CH}_{2} \mathrm{CH}_{2}\right.$ ), 3.30-3.21 (m, 1H, ArCHCH $)_{2}, 3.77-3.69\left(\mathrm{~m}, 1 \mathrm{H}, \mathrm{ArCHCH}_{2}\right), 3.87$ (s, $3 \mathrm{H}, o-\mathrm{CH}_{3} \mathrm{O}$ ), $3.89\left(\mathrm{~s}, 3 \mathrm{H}, p-\mathrm{CH}_{3} \mathrm{O}\right), 4.23(\mathrm{~d}, J=11.6 \mathrm{~Hz}, 1 \mathrm{H}$, $\left.\mathrm{ArCH}_{2}\right), 4.38\left(\mathrm{dd}, J=8.8 \mathrm{~Hz}, J=3.8 \mathrm{~Hz}, 1 \mathrm{H}, \mathrm{ArCHCH}_{2}\right)$, $4.48\left(\mathrm{~d}, J=11.6 \mathrm{~Hz}, 1 \mathrm{H}, \mathrm{ArCH}_{2}\right), 5.11\left(\mathrm{~s}, 2 \mathrm{H}, \mathrm{ArCH}_{2}\right), 5.83$ (br, $1 \mathrm{H}, \mathrm{CONH}), 6.84-6.74(\mathrm{~m}, 3 \mathrm{H}, \mathrm{Ar}-\mathrm{H}), 7.02(\mathrm{~d}, J=8.4 \mathrm{~Hz}$, 2H, Ar-H), 7.46-7.25 (m, 8H, Ar-H), 7.48 (d, J = 7.4 Hz, 2H, Ar-H). HRMS (ESI) m/z Calcd for $\mathrm{C}_{33} \mathrm{H}_{35} \mathrm{NO}_{5} \mathrm{Na}^{+}[\mathrm{M}+\mathrm{Na}]^{+}$ 548.2407, found 548.2405.

N-(2-(4-(Benzyloxy)phenyl)-2-(prop-2-yn-1-yloxy)ethyl)-3(3,4-dimethoxyphenyl)propanamide $4 f$. White solid, yield $68 \%$, m.p. $93-94^{\circ} \mathrm{C},{ }^{1} \mathrm{H}$ NMR $\left(400 \mathrm{MHz}, \mathrm{CDCl}_{3}\right) \delta 2.41$ $(\mathrm{s}, 1 \mathrm{H}, \mathrm{C} \equiv \mathrm{CH}), 2.48\left(\mathrm{t}, J=7.6 \mathrm{~Hz}, 2 \mathrm{H}, \mathrm{CH}_{2} \mathrm{CH}_{2}\right), 2.92$ $\left(\mathrm{t}, J=7.6 \mathrm{~Hz}, 2 \mathrm{H}, \mathrm{CH}_{2} \mathrm{CH}_{2}\right), 3.13-3.20\left(\mathrm{~m}, 1 \mathrm{H}, \mathrm{CH}_{2} \mathrm{CH}\right)$, 3.70-3.78 (m, $\left.1 \mathrm{H}, \mathrm{CH}_{2} \mathrm{CH}\right), 3.82\left(\mathrm{~d}, J=2.0 \mathrm{~Hz}, 1 \mathrm{H}, \mathrm{OCH}_{2}\right)$, $3.86\left(\mathrm{~s}, 3 \mathrm{H}, o-\mathrm{ArOCH}_{3}\right), 3.88\left(\mathrm{~s}, 3 \mathrm{H}, p-\mathrm{ArOCH}_{3}\right), 4.08$ (d, $\left.J=2.0 \mathrm{~Hz}, 1 \mathrm{H}, \mathrm{OCH}_{2}\right), 4.47(\mathrm{dd}, J=7.6 \mathrm{~Hz}, J=9.2 \mathrm{~Hz}$, $1 \mathrm{H}, \mathrm{CH}_{2} \mathrm{CH}$ ), 5.06 (s, 2H, p-ArOCH${ }_{2} \mathrm{Ph}$ ), 5.87 (br, $1 \mathrm{H}, \mathrm{NH}$ ), 6.74-6.81 (m, 3H, Ar-H), 6.96 (d, J = 8.8 Hz, 2H, Ar-H), 7.21 (d, $J=8.8 \mathrm{~Hz}, 2 \mathrm{H}, \mathrm{Ar}-\mathrm{H}$ ), 7.32-7.44 (m, 5H, Ar-CH). HRMS (ESI) $\mathrm{m} / \mathrm{z}$ Calcd for $\mathrm{C}_{29} \mathrm{H}_{31} \mathrm{NO}_{5} \mathrm{Na}^{+}[\mathrm{M}+\mathrm{Na}]^{+}$496.2094, found 496.2090.

N-(2-(Allyloxy)-2-(4-(benzyloxy)phenyl)ethyl)-3-(3,4-dimethoxyphenyl)propanamide $\mathbf{4 g}$. White solid, yield $65 \%$, m.p. $68-69^{\circ} \mathrm{C},{ }^{1} \mathrm{H}$ NMR $\left(400 \mathrm{MHz}, \mathrm{CDCl}_{3}\right) \delta 2.57-2.41(\mathrm{~m}, 2 \mathrm{H}$, $\left.\mathrm{CH}_{2} \mathrm{CH}_{2}\right), 2.92\left(\mathrm{~d}, \mathrm{~J}=7.1 \mathrm{~Hz}, 2 \mathrm{H}, \mathrm{CH}_{2} \mathrm{CH}_{2}\right), 3.38-3.15(\mathrm{~m}$, $\left.2 \mathrm{H}, \mathrm{CH}_{2} \mathrm{CH}\right), 3.78-3.58\left(\mathrm{~m}, 2 \mathrm{H}, \mathrm{CH}_{2} \mathrm{CH}\right), 3.97-3.81(\mathrm{~m}, 6 \mathrm{H}$, $\left.p-\mathrm{CH}_{3} \mathrm{O}+o-\mathrm{CH}_{3} \mathrm{O}\right), 4.74\left(\mathrm{~d}, J=7.8 \mathrm{~Hz}, 1 \mathrm{H}, \mathrm{CH}_{2} \mathrm{CH}\right), 5.07$ (s, $\left.1 \mathrm{H}, \mathrm{CH}_{2} \mathrm{CH}\right), 5.20(\mathrm{dd}, J=21.0 \mathrm{~Hz}, J=13.9 \mathrm{~Hz}, 1 \mathrm{H}$, $\mathrm{CHPh}), 5.85$ (br, $1 \mathrm{H}, \mathrm{CONH}), 6.87-6.70\left(\mathrm{~m}, 3 \mathrm{H}, \mathrm{CH}=\mathrm{CH}_{2}\right)$, 6.96 (s, 1H, Ar-H), 7.53-7.16 (m, 7H, Ar-H). HRMS (ESI) $\mathrm{m} / \mathrm{z}$ Calcd for $\mathrm{C}_{29} \mathrm{H}_{33} \mathrm{NO}_{5} \mathrm{Na}^{+}[\mathrm{M}+\mathrm{Na}]^{+}$498.2251, found 498.2258 . 


\subsection{Antiviral Activity}

3.3.1. Purification of TMV. Using Gooding's method [9], the upper leaves of Nicotiana tabacum L. inoculated with TMV were selected and ground in phosphate buffer and then filtered through double-layer pledget. The filtrate was centrifuged at $10000 \mathrm{~g}$, treated with PEG twice, and centrifuged again. The whole experiment was processed at $4^{\circ} \mathrm{C}$. Absorbance value was estimated at $260 \mathrm{~nm}$ by ultraviolet spectrophotometer. Consider

$$
\text { virus concn }=\frac{\left(A_{260} \times \text { dilution ratio }\right)}{E_{1 \mathrm{~cm}}^{0.1 \%, 260 \mathrm{~nm}}}
$$

3.3.2. Preparation of Medicaments. Tested compounds and ribavirin used as a reference antiviral agent were first dissolved in minimum volume of N,N-dimethylformamide (DMF) and then diluted with distilled water containing $1 \%$ Tween 20 at $500 \mu \mathrm{g} / \mathrm{mL}$ concentration.
3.4. Protection Effect of Compounds against TMV In Vivo. The compound solution was smeared on the left side and the solvent serving as control on the right side of growing $N$. tabacum L. leaves of the same ages. The leaves were then inoculated with the virus after $12 \mathrm{~h}$. A brush was dipped in TMV of $6 \times 10^{-3} \mathrm{mg} / \mathrm{mL}$ to inoculate the leaves, which were previously scattered with silicon carbide. The leaves were then washed with water and rubbed softly along the nervature once or twice. The local lesion numbers appearing 3-4 days after inoculation were counted [10]. There are three replicates for each compound.

3.5. Curative Effect of Compounds against TMV In Vivo. The growing leaves of Nicotiana tabacum L. of the same ages were selected. The tobacco mosaic virus (concentration of $6 \times 10^{-3} \mathrm{mg} / \mathrm{mL}$ ) was dipped and inoculated on the whole leaves. The leaves were then washed with water and dried. The compound solution was smeared on the left side, and the solvent was kept on the right side for control. The local lesion numbers were then recorded 3-4 days after inoculation [11]. For each compound, three repetitions were conducted to ensure the reliability of the results, which were measured according to the following formula:

$$
\text { inhibition rate }(\%)=\frac{\text { av local lesion numbers of control (not treated with compound) av local lesion numbers smeared with drugs }}{\text { av local lesion numbers without drugs }} \text {. }
$$

\section{Conflict of Interests}

The authors declare that they have no conflict of interests.

\section{Acknowledgments}

The authors are grateful to the financial support for this work from the National Natural Science Foundation of China (21172124), the National Basic Research Science Foundation of China (2010CB126105), and the National Key Technologies R\&D Program (2011BAE06B05).

\section{References}

[1] O. A. Postnikova and L. G. Nemchinov, "Comparative analysis of microarray data in Arabidopsis transcriptome during compatible interactions with plant viruses," Virology Journal, vol. 9, p. 101, 2012.

[2] W. Li, N. Li, Y. Tang et al., "Biological activity evaluation and structure-activity relationships analysis of ferulic acid and caffeic acid derivatives for anticancer," Bioorganic \& Medicinal Chemistry Letters, vol. 22, pp. 6085-6088, 2012.

[3] S. Balakrishnan, V. P. Menon, and S. Manoharan, "Ferulic acid inhibits 7,12-dimethylbenz $[\alpha]$ anthracene-induced hamster buccal pouch carcinogenesis," Journal of Medicinal Food, vol. 11, no. 4, pp. 693-700, 2008.
[4] E. Y. Kwon, G. M. Do, Y. Y. Cho, Y. B. Park, S. M. Jeon, and M. S. Choi, "Anti-atherogenic property of ferulic acid in apolipoprotein E-deficient mice fed Western diet: comparison with clofibrate," Food and Chemical Toxicology, vol. 48, no. 8-9, pp. 2298-2303, 2010.

[5] X. F. Lin, W. Min, and D. Luo, "Anticarcinogenic effect of ferulic acid on ultraviolet-B irradiated human keratinocyte HaCaT cells," Journal of Medicinal Plant Research, vol. 4, no. 16, pp. 1686-1694, 2010.

[6] H. Shanmugam and M. Doble, "Combination of ferulic acid and antibiotics as effective antibacterial agents," Planta Medica, vol. 76, no. 12, p. 1191, 2010.

[7] T. Tetsuka, L. D. Baier, and A. R. Morrison, "Antioxidants inhibit interleukin-1-induced cyclooxygenase and nitric-oxide synthase expression in rat mesangial cells: evidence for posttranscriptional regulation," Journal of Biological Chemistry, vol. 271, no. 20, pp. 11689-11693, 1996.

[8] L. Ou, L. Y. Kong, X. M. Zhang, and M. Niwa, "Oxidation of ferulic acid by Momordica charantia peroxidase and related antiinflammation activity changes," Biological and Pharmaceutical Bulletin, vol. 26, no. 11, pp. 1511-1516, 2003.

[9] G. V. Gooding Jr. and T. T. Hebert, "A simple technique for purification of tobacco mosaic virus in large quantities," Phytopathology, vol. 57, no. 11, p. 1285, 1967.

[10] K. Wang, B. O. Su, Z. Wang et al., "Synthesis and antiviral activities of phenanthroindolizidine alkaloids and their derivatives," Journal of Agricultural and Food Chemistry, vol. 58, no. 5, pp. 2703-2709, 2010. 
[11] X. Gao, X. Cai, K. Yan, B. Song, L. Gao, and Z. Chen, "Synthesis and antiviral bioactivities of 2-aryl- or 2-methyl-3-(substitutedbenzalamino)-4(3H)-quinazolinone derivatives," Molecules, vol. 12, no. 12, pp. 2621-2642, 2007. 

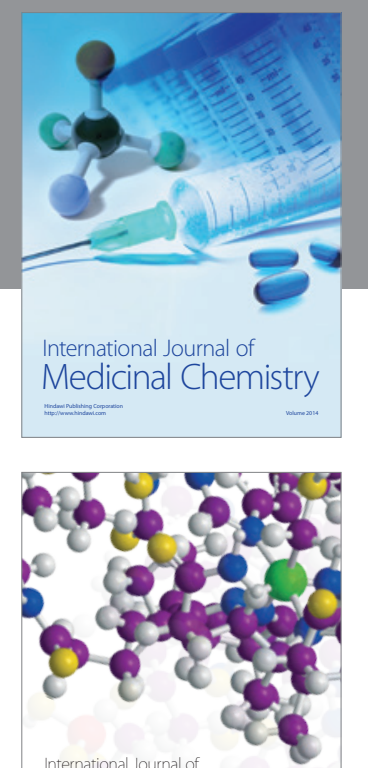

\section{Carbohydrate} Chemistry

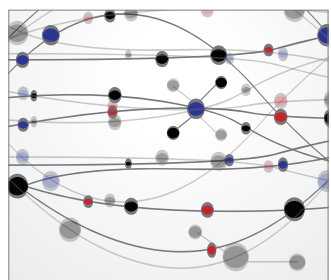

The Scientific World Journal
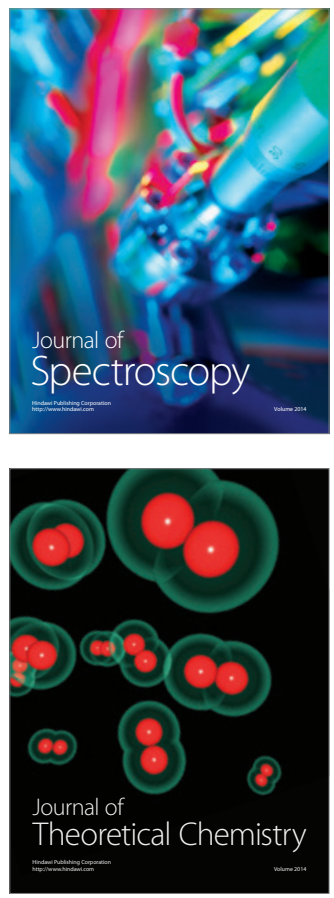
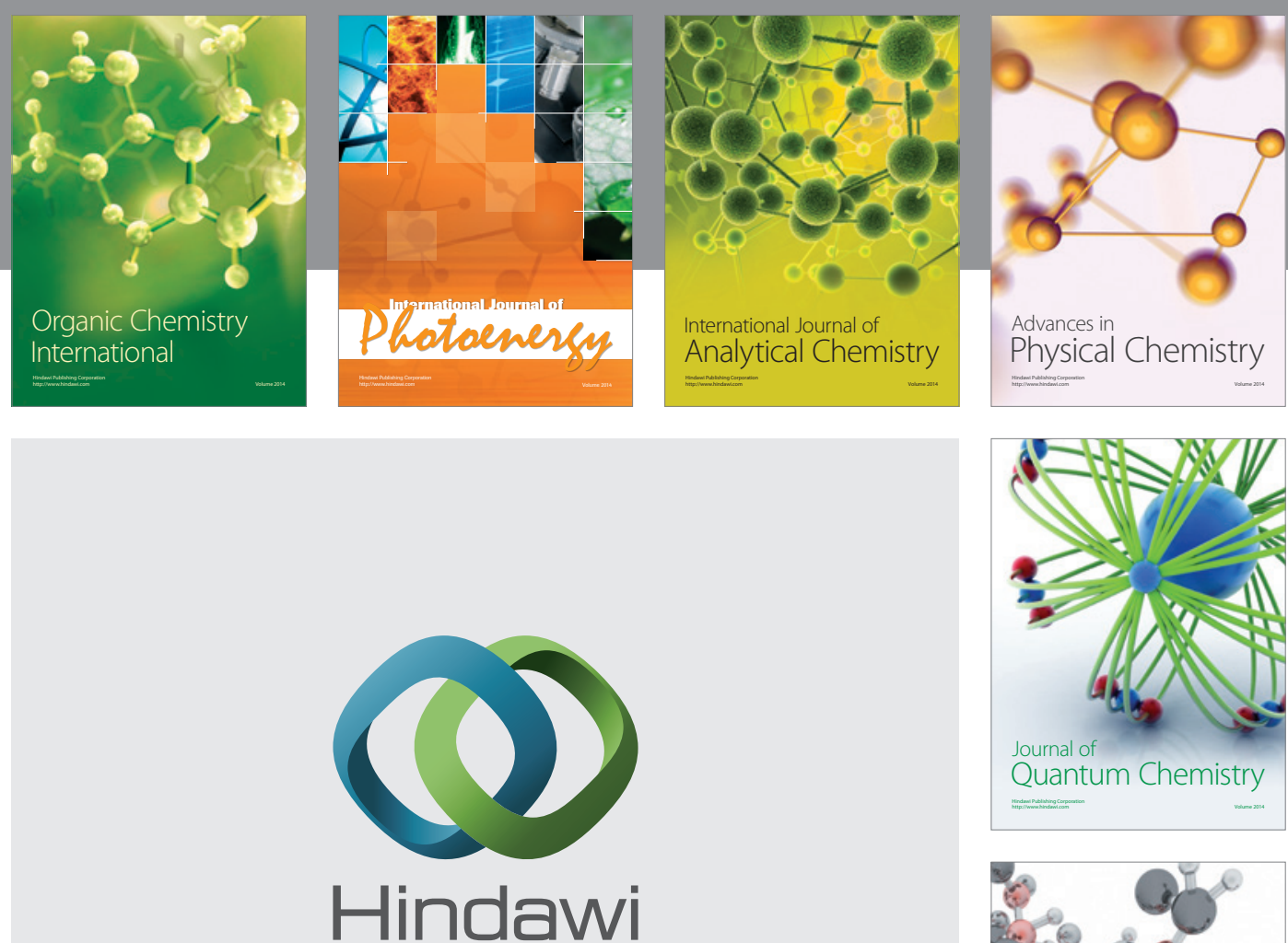

Submit your manuscripts at

http://www.hindawi.com

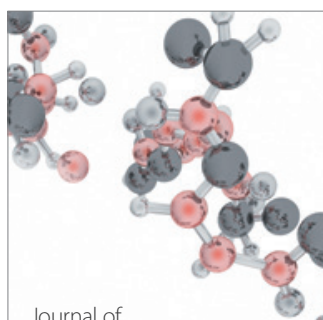

Analytical Methods

in Chemistry

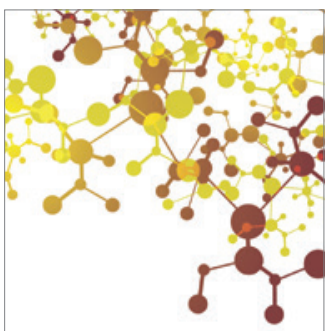

Journal of

Applied Chemistry

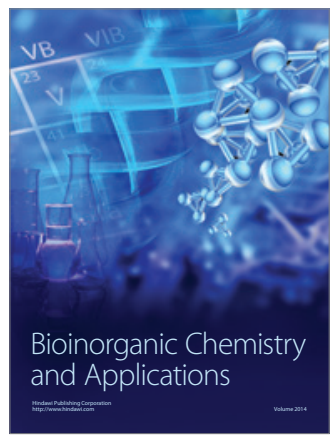

Inorganic Chemistry
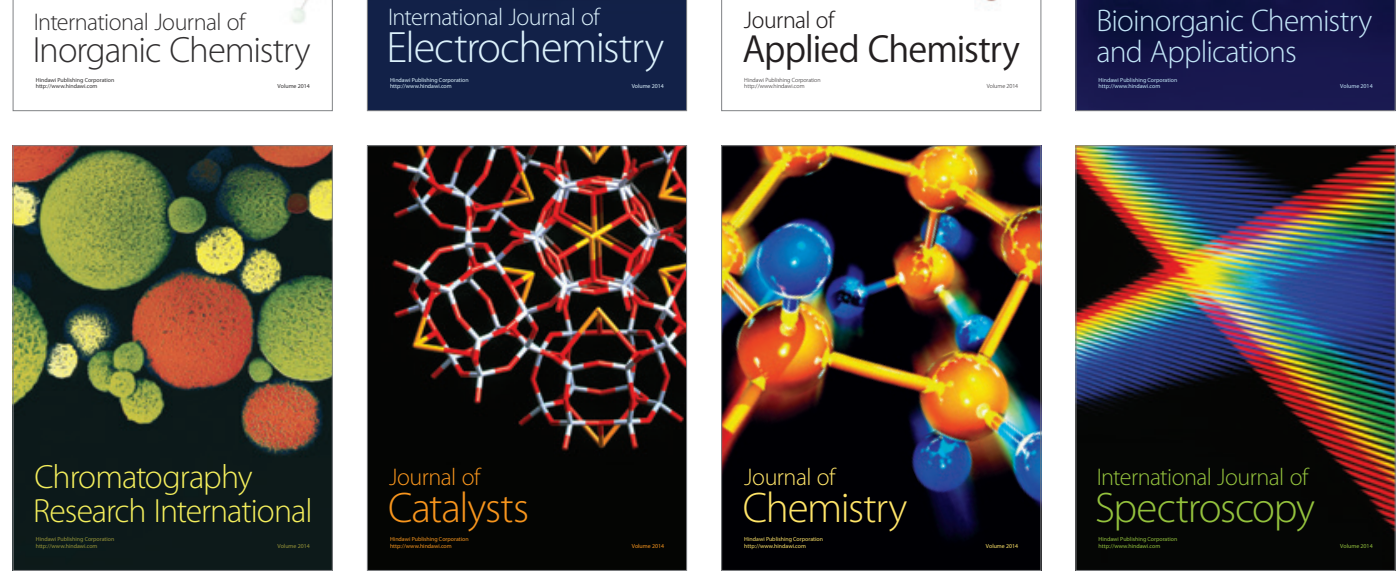